\title{
Performance Evaluation: Worth the Cost?
}

\author{
John Lubans, Jr.
}

Evaluation: The most important activity conducted in an organization between employee and supervisor. Probably of all their work, the least understood and most poorly done in the least amount of time.

This anonymous definition may have crossed our minds in cynical moments as we struggled with performance evaluations, both in giving them and accepting them. Of course, the appraisal process is seen as necessary by most; in fact, evaluation or appraisal of library work is something with which librarians deal daily. Usually, mordant comments like the above definition arise from the (for some) anxiety-laden application of a formal, written performance evaluation system at a specific time of the year in which both the supervised and supervisors take part. Suggesting the profession's preoccupation with evaluation are the numerous examples of forms used prior to, during, and after the face-to-face meeting. These range from exhaustive checklists of desirable/undesirable traits to lengthy narrative statements, by librarians and department heads, summing up the year's work. In one example, a total of eight signatures and counter-signatures (from line librarian to director) are required of the participants.

This article examines the complexities of the personnel evaluation process in libraries as a service industry and asks whether or not it is worth the price paid in time and, in some cases, frustration by the participants.

\section{What happens in the evaluation process?}

The participants do not come with a blank slate to this annual rite. Ideally, a regular, even daily, exchange of information has occurred during the work of the library. Often there has been some goal setting in the past, and the annual evaluation is meant to discuss how well such goals have been met or even exceeded.

John Lubans, Jr., is Assistant University Librarian for Public Services at Duke University.
This expectation of more can represent a major sensitive area. Most mature organizations have well-established, routine subsystems such as, in libraries, cataloging and reference. Unlike developing or new organizations with undefined boundaries, libraries have few areas in which disruptive, constant change prevails. Traditional goals are often tacitly understood; a job well done may not vary from year to year. Too often, we may be led to expect an increase in productivitymore books processed, more questions answered, more budget dollars - to verify growth. When this incrementalism does not occur, qualitative judgments must come into play, or we risk creating artificial increases in order to satisfy the evaluation process and thereby pervert it. Instead, while "production" may remain the same, we often say that we are doing it better, that there are fewer errors and more satisfied customers. Obviously, this can lead to evaluation difficulties. Lacking the numerical data that suggest growth, we are faced, with the time-consuming challenge of explaining and understanding the subtle improvements made from year to year.

The greatest flaw in formal evaluation may be found in the lack of communication among the participants. Evaluating professionals is a difficult process because often the evaluator, who has other responsibilities besides evaluation, may not be familiar with the daily, specialized routines of a particular person. A new program such as a computer data base reference service can serve as an example. How is an administrator who is admittedly a computer illiterate to evaluate the highly specialized performance of the professional providing this service? Obviously, information must be exchanged. It should be noted here that the expression of mutual expectations and awareness is the responsibility of both sides in the evaluation process. The absence of this exchange may lead to the frustration underlying the cynicism in the introductory definition.

In the service industry, the customer (or user) is the person we seek to satisfy. While we may know the numbers of library users and uses made, we generally lack information about the 
user's perception of the quality of our services. Attempts to measure these perceptions are made from time to time through subjective observation and the practical but imperfect user survey. In administrative theory, the customer can be viewed as part of the organization. If we subscribe to this idea, we have yet to establish effective channels for gaining from this group feedback of value in performance appraisal.

Another feature of mature professional organizations like libraries is a stable workforce with limited turnover. It is often the same people who do the good (and in some cases, the not-so-good) job from year to year. Market forces may also influence management's expectations of the line employee. If a surplus of librarians exists, economics dictate that more can be expected of each employee, and vice versa. On the other hand, regardless of market conditions, the individual worker may seek to achieve job security and, having done so through unions, tenure, or civil service, may be able to resist calls for greater production or participation in new programs. The morality of this approach is beside the point; it is an economic reality that operates at all organizational levels. Evaluating performance under these circumstances can be trying (some would say challenging), since a supervisor's expectations may exceed locally acceptable performance norms.

Another variable influencing the participants is the organizational "culture." This mix of factors, ranging from dress codes to parking lot privileges, will either hinder or facilitate the evaluation process. The more open and informal the culture, the better the chance that a nonthreatening exchange may take place.

In my view, "professionalism" is the leading contributor to the complexity of personnel evaluation in academic libraries. Frequently, evaluation systems have evolved concurrently with the librarian's pursuit of academic or faculty status. Many campuses have granted their librarians both full faculty ranking and some version of the faculty evaluation process. Within such systems the professional aspect of our work was expanded (voluntarily) to include, in generous portions, not only the completion of job responsibilities but also research and publication and professional, societal service. This trend now appears to have run its course, and the heady delight of being listed in the campus directory as an assistant professor has now been turned into the insecure reality of having to publish or perish and to do without work time alloted for this purpose. Because of this unexpected turn of events, former advocates of what they perceived as higher professional status now may be overcompensating.

While any definition of library professionalism would surely include the types of things our faculty colleagues do that relate to our work, there is some concern as to how much, if any, of our time should be spent on extracurricular activities for consideration in performance appraisal. Some supervisors may use the presence or absence of such activities to differentiate among individual performances. This uncertainty of what a professional does has led to some confusion between the two groups-the evaluator and the evaluated.

Professionalism also accounts for the different emphasis that each librarian gives a position. I have suggested elsewhere ${ }^{1}$ an observed philosophical dichotomy among librarians: there are those who subscribe to the use ethos and those who subscribe to the conservation ethos. One emphasizes collection over service. Neither denies the value of the other. It is a matter of priorities. In the evaluation process, conflict can erupt over the emphasis placed by either of the parties on certain ones of multiple duties. The acceptance of these philosophical differences is important to the evaluation process. However, organizational and program emphasis may mandate a change which is not in concert with an individual librarian's philosophical stance. For example, a reduction in the quality of original cataloging or in the quality/quantity of reference service may be abhorrent to an individual professional. The librarian is then faced with resisting, changing the organization, or leaving.

Some people view evaluation as impossibly difficult because of the lack of hard and fast quantities (also known as standards). They point enviously to the for-profit sector with its renowned "bottom line" as the common denominator of all its evaluations. However, a bit of pondering results in the realization that even in business a multitude of factors come into play in the evaluation process. The fact that this year's sale of cheeseburgers is 30 per cent off last year's figure will rarely result in the firing of a fast food store's manager. Very likely, the "bottom line" is only one of several considerations in the evaluation of individual and corporate performance. Just as retrenchment is prevalent among libraries and is used to justify reduction in service, so can economic downturns, recessions, and the like be used in similar fashion in the for-profit sector. Likewise, comparable communication and group processes occur in profit and nonprofit groups. Decisions are made, memos are exchanged, meet- 
ings are convened and chaired, supervisors supervise, and managers manage. People working with other people tend to encounter the same difficulties-unfocused goals, worker dissatisfaction-regardless of their public or private employer.

Given this, the circle of those of us with mutual concerns about performance evaluation should be widened. At the same time, no ready answers are to be found in the for-profit sector, only questions similar to the ones we already have. Indeed, it might even be said that not having a profit or loss column in our measurements of performance actually provides a less threatening climate for evaluation.

\section{Is it worth it?}

All organizations, regardless of their size, demand communication among their members. Formal evaluation systems are designed to encourage the exchange of ideas and questions among levels of responsibility. The process is important in realizing organizational goals, stated or implied. In some ways, an annual performance evaluation is a form of "checks and balances" in the organization. It can reveal whether or not all members are working toward similar goals. If not, then theoretically the evaluation process can be used to motivate individuals and/or change their direction.

It should be remembered that as we evaluate parts of the organization, we essentially evaluate the health of the total organization. Conversely, it is sometimes too easy to forget that the ills of the organization (a city, a university campus, a school district) may underlie what are perceived as flaws in an individual.

Common to libraries and the for-profit sector is the question of how to motivate, through the evaluation process, the fair-to-good performer. Without the flow of communication demanded by the evaluation channel, such motivation could be left to chance. No doubt there is a lack of any quantitative evidence that the evaluation process results in improvements, however gradual, among this group of individuals, but one suspects that it does result in such improvements if the evaluation is done and done fairly and consistently. While there may be some uncertainty about the effect of evaluation on the fair-to-good performer, there is less doubt about its effect on the extremes, those individuals performing at unacceptable levels or those exceeding expectations. The latter need the recognition which is a form of recompense for performing better than others and which is furthermore the positive feedback that should serve to encourage maintenance of this level of performance. (At least, evaluation should not harm this group.) For the former, evaluation serves as documentation of unmet expectations and as a guideline for improvement. It is a part of due process, should it come to that. Evaluation here is helpful in that it can bring supervisor and employee together to discuss what otherwise, because of the human condition, might be (and often is) avoided. It assures, however perfunctorily, the exchange of information about what is expected and how improvements can be made. In some cases, the individual can then be guided to higher levels of performance. For others, separation proceedings will not be a surprise.

In summary, performance evaluation is difficult and complex, but when done well it strengthens and affirms the positive contributions most of us make as professionals, recognizes the value of our role in the organization, and provides consistency in our services through the sharing of organizational goals and expectations. As a colleague aptly remarked, no one likes to be judged, but all expect others to be judged so that they can improve. If performance evaluation is done consistently throughout the year, it can be a source of organizational well-being.

\section{References}

1. John Lubans, Jr., "Teaching the User: Ethical Considerations," Reference Librarian 4 (Summer 1982):89-98. 